\title{
Einfluss des Body-Mass-Index auf den Erfolg nach Trabektomoperation?
}

\section{Body Mass Index as an Influencing Factor for Outcome of Trabectome Surgery?}

Autoren

Institut
A. Anton, M. Neuburger, T. Wecker, D. Böhringer, J. F. Jordan

Klinik für Augenheilkunde, Universitätsklinikum Freiburg

Schlüsselwörter
Glaukom
Trabektom
Body-Mass-Index
episkleraler Venendruck
Liquordruck
Key words
glaucoma
trabectome
body mass index
cerebrobrospinal fluid pressure

eingereicht 11.2.2014 akzeptiert 28.4.2014

Bibliografie

Dol http://dx.doi.org/ 10.1055/s-0034-1368576 Online-publiziert 12.9.2014 Klin Monatsbl Augenheilkd 2014; 231: 1103-1106 (c) Georg Thieme Verlag KG Stuttgart . New York · ISSN 0023-2165

\section{Korrespondenzadresse} Dr. Alexandra Anton, MD Universitätsklinikum Freiburg Klinik für Augenheilkunde Killianstraße 5 79106 Freiburg Tel.: $0761 / 27040010$ Fax: 0761/27041030 alexandra.anton@uniklinikfreiburg.de

\section{Zusammenfassung \\ $\nabla$}

Einleitung: Ziel der Studie war es, einen möglichen Einfluss des Body-Mass-Index (BMI) auf das Ergebnis nach Trabektomoperation zu untersuchen.

Patienten und Methoden: 131 Augen mit primärem Offenwinkelglaukom (POWG), Myopieglaukom (MG) und Pseudoexfoliationsglaukom (PEXG), bei denen die Indikation zur Trabektomchirurgie gestellt wurde, wurden in diese retrospektive Studie eingeschlossen. Die Daten wurden der Freiburger Trabektomdatenbank von Juni 2009 bis April 2013 entnommen. Mittels eines Cox-Proportional-Hazards-Modells wurde der Einfluss des BMI auf das Operationsergebnis unter Berücksichtigung verschiedener Kovariaten (Alter, präoperative Tropftherapie) berechnet.

Ergebnis: Die Gruppe mit einem BMI $>25 \mathrm{~kg} / \mathrm{m}^{2}$ weist ein statistisch signifikant schlechteres Ergebnis bezogen auf den absoluten Erfolg (20\% Drucksenkung vom Ausgangsdruck ohne drucksenkende Medikation) nach Trabektomoperation auf $(\mathrm{p}=0,047)$. Hinsichtlich des relativen Erfolgs sowie der Reoperationsrate waren keine statistisch signifikanten Unterschiede zu beobachten.

Schlussfolgerung: In unserer Patientenkohorte von 131 Augen war ein hoher BMI mit einer reduzierten Erfolgsaussicht assoziiert, solange ein absoluter Erfolg gefordert wird. Sind Medikamente akzeptabel (relativer Erfolg), ist kein Unterschied zu beobachten.

\section{Einleitung \\ $\nabla$}

Die Anzahl der übergewichtigen Patienten steigt in den letzten Jahrzehnten stetig an. Seit 1980 nimmt der BMI weltweit jedes Jahrzehnt um $0,4 \mathrm{~kg} / \mathrm{m}^{2}$ zu und stellt so ein zunehmendes Problem für die Sozialsysteme in Industriestaaten dar [1]. Viele Erkrankungen, wie koronare Herzkrank-

\section{Abstract \\ $\nabla$}

Background: The aim of this study was to investigate a possible influence of body mass index (BMI) to the outcome of trabectome surgery.

Methods: 131 eyes with primary open angle glaucoma, myopia-associated glaucoma and pseudoexfoliation glaucoma were included into this retrospective study. The data were extracted from the Freiburg trabectome database from June 2009 to April 2013. We fitted a Cox proportional hazards model in order to assess the influence of the BMI on trabectome outcome.

Results: The absolute success after trabectome surgery (20\% pressure reduction without antiglaucomatous medication) was statistically significantly better in the group with BMI $>25 \mathrm{~kg} / \mathrm{m}^{2}$ $(p=0.047)$. No statistically significant effect was observed for relative success or the rate of re-operation respectively.

Conclusion: In our patient cohort of 131 eyes, a high BMI was associated with a reduced success, as long as an absolute success is required. No difference is seen if additional anti-glaucomatous medication is acceptable (relative success).

heit oder Diabetes mellitus Typ 2, können auf Übergewicht zurückgeführt werden [2].

Eine Hochrechnung weltweiter epidemiologischer Daten zum Glaukom zeichnet voraus, dass im Jahre 2020 voraussichtlich 79,6 Millionen Menschen am Glaukom leiden werden, hiervon werden schätzungsweise 11,2 Millionen Menschen bereits an beiden Augen erblindet sein [3] 
Tab. 1 Basisdaten der eingeschlossenen Patienten. Die beiden Gruppen unterschieden sich nicht hinsichtlich der Glaukomform, des Alters, des Ausgangsdrucks und der präoperativen Tropftherapie voneinander.

\begin{tabular}{|c|c|c|c|}
\hline & $\begin{array}{l}\text { BMI: } \leq 25 \mathrm{~kg} / \mathrm{m}^{2} \\
(\mathrm{n}=62)\end{array}$ & $\begin{array}{l}\text { BMI: > } 25 \mathrm{~kg} / \mathrm{m}^{2} \\
(\mathrm{n}=69)\end{array}$ & \multirow{4}{*}{$p=0,65$} \\
\hline MG in Prozent (Anzahl) & $8 \%(5)$ & $13 \%(9)$ & \\
\hline PEXG in Prozent (Anzahl) & $34 \%(21)$ & $32 \%(22)$ & \\
\hline POWG in Prozent (Anzahl) & $58 \%(36)$ & $55 \%(38)$ & \\
\hline Alter (in Jahren) & $66 \pm 13$ & $67 \pm 12$ & $p=0,19$ \\
\hline Geschlecht m: w & $34: 66 \%(21: 41)$ & $42: 58 \%(29: 40)$ & \\
\hline Anzahl der präoperativen Augentropfen & $2,1 \pm 1,3$ & $2,2 \pm 1,4$ & $p=0,45$ \\
\hline IOD präoperativ in mmHG & $25,6 \pm 6,4$ & $24,1 \pm 5,5$ & $p=0,12$ \\
\hline Nachbeobachtungszeitraum in Tagen & $360 \pm 356$ & $387 \pm 352$ & $p=0,49$ \\
\hline
\end{tabular}

MG: Myopieglaukom, PEXG: Pseudoexfoliationsglaukom, POWG: primäres Offenwinkelglaukom, IOD: intraokularer Druck, BMI: Body-Mass-Index

Somit wird auch die Zahl übergewichtiger Glaukompatienten überproportional ansteigen.

Ein indirekter Zusammenhang zwischen Übergewichtigkeit und Glaukom wird vermutet. Mehrere Studien haben gezeigt, dass ein hoher BMI mit einem höheren Augeninnendruck (IOD) assoziiert ist [4-7]. Bei Übergewichtigen liegt ein erhöhter episkleraler Venendruck vor [7-9], der über eine Erhöhung des Abflusswiderstands zu einer Erhöhung des IOD führt. Erklären ließe sich dies einerseits durch vermehrtes orbitales Fettgewebe bei Patienten mit erhöhtem BMI und damit indirekt einem reduzierten Abfluss des Kammerwassers über das episklerale Venensystem durch Kompression desselben [7]. Andererseits liegt bei Patienten mit einem erhöhtem BMI ein erhöhter intraabdomineller Druck vor [8], der über einen erhöhten Druck auf das venöse Gefäßsystem ebenfalls zu einen Abflussstörung über das episklerale Venensystem führt.

Vor diesem Hintergrund haben wir einen möglichen Einfluss des Body-Mass-Index (BMI) auf das Ergebnis nach Trabekulotomie ab interno mit dem Trabektom untersucht. Die Trabektomoperation ist ein minimalinvasiver Eingriff mit einem günstigen Komplikationsprofil und daher gerade bei frühen Glaukomformen gut einsetzbar. Bei der Trabektomoperation wird das Trabekelmaschenwerk, welches den Hauptabflusswiderstand beim Glaukom darstellt, über 3-5 Uhrzeiten mittels Elektroablation entfernt, sodass das Kammerwasser direkt zur skleralen Wand mit den Kollektorkanälen hin abfließen kann (da nun eine direkte Verbindung zwischen Kollektorkanälen und Vorderkammer besteht). Durch die Entfernung des Hauptabflusswiderstands kommen hier möglicherweise den weiter posterior des Trabekelmaschenwerks gelegenen 3-dimensional verzweigten Abflusswegen [10,11] vermehrt Bedeutung zu. Ein bei Übergewichtigen erhöhten episkleraler Venendruck [7-9] könnte zu einem erhöhten Abflusswiderstand und somit zu einem schlechteren Ergebnis nach Trabektomoperation führen. Um diese Hypothese zu überprüfen, haben wir eine retrospektive Analyse unserer Trabektomdatenbank durchgeführt und die 3 häufigsten Glaukomformen - primäres Offenwinkelglaukom (POWG), Myopieglaukom (MG) und Pseudoexfoliationsglaukom (PEXG) - untersucht.

\section{Patienten und Methoden}

$\nabla$

Eingeschlossen wurden alle Patienten, bei denen im Zeitraum von Juni 2009 bis April 2013 in der Universitätsaugenklinik Freiburg eine Trabektomoperation durchgeführt worden ist und die als POWG, MG oder Pseudoexfoliationsglaukom PEXG klassifi- ziert wurden. Ausgeschlossen waren alle Patienten, die eine kombinierte Katarakt-/Trabektomoperation erhielten, da die additive Kataraktoperation einen Einfluss auf das Ergebnis nach Trabektomoperation zu haben scheint [12].

Die Operationen wurden von 2 erfahrenen Operateuren (MN, JJ) durchgeführt.

Alle Patienten wurden direkt am Tag nach der Operation sowie 3 Monate postoperativ untersucht. Bei jedem weiteren Besuch des Patienten in unserer Klinik wurden der aktuelle Druck sowie die aktuelle Therapie elektronisch erfasst. Die Entscheidung zur Reoperation wurde klinisch, individuell festgelegt. Es gab keine prospektiv festgelegten Erfolgskriterien.

Als primärer Endpunkt wurde eine 20\%ige Drucksenkung ohne additive drucksenkende Therapie definiert. Als sekundäre Endpunkte wurden eine 20\%ige Drucksenkung mit additiver drucksenkender Therapie und eine Reoperation definiert.

Insgesamt wurden 131 Augen in die statistische Auswertung eingeschlossen.

Eine Einwilligung der Patienten zur Auswertung der Daten liegt vor. Ein Votum der zuständigen Ethikkommission der AlbertLudwigs-Universität Freiburg liegt vor (Nr.: 235/10_160678). Die 131 Augen wurden in 2 Gruppen unterteilt (übergewichtig: $>25 \mathrm{~kg} / \mathrm{m}^{2}$, unter-/normalgewichtig $\leq 25 \mathrm{~kg} / \mathrm{m}^{2}$, Einteilung laut WHO-Klassifikation). Auf eine weitere Unterteilung in eine zusätzliche Gruppe der Untergewichtigen wurde hinsichtlich der Fragestellung sowie der geringen Anzahl der Untergewichtigen $(n=6)$ verzichtet. Hinsichtlich möglicher Einflussfaktoren wie Glaukomtyp, Ausgangsdruck, Alter oder präoperative Tropftherapie unterschieden sich die Gruppen statistisch nicht (siehe - Tab. 1).

\section{Statistik \\ $\nabla$}

Mittels eines Cox-Proportional-Hazards-Modells wurde der Einfluss des BMI auf das Überschreiten der postoperativen Druckwerte von $80 \%$ des Ausgangsdrucks ( $\triangleq$ IOD $<-20 \%$ ) ohne medikamentöse Therapie (absoluter Erfolg) bzw. mit medikamentöser Therapie (relativer Erfolg) untersucht. Zusätzlich wurde die Versagensquote (= Durchführung einer weiteren drucksenkenden Operation) berechnet.

In diesem Modell wurden die folgenden Kovariaten berücksichtigt: Alter zum Operationszeitpunkt und präoperative Tropftherapie. 


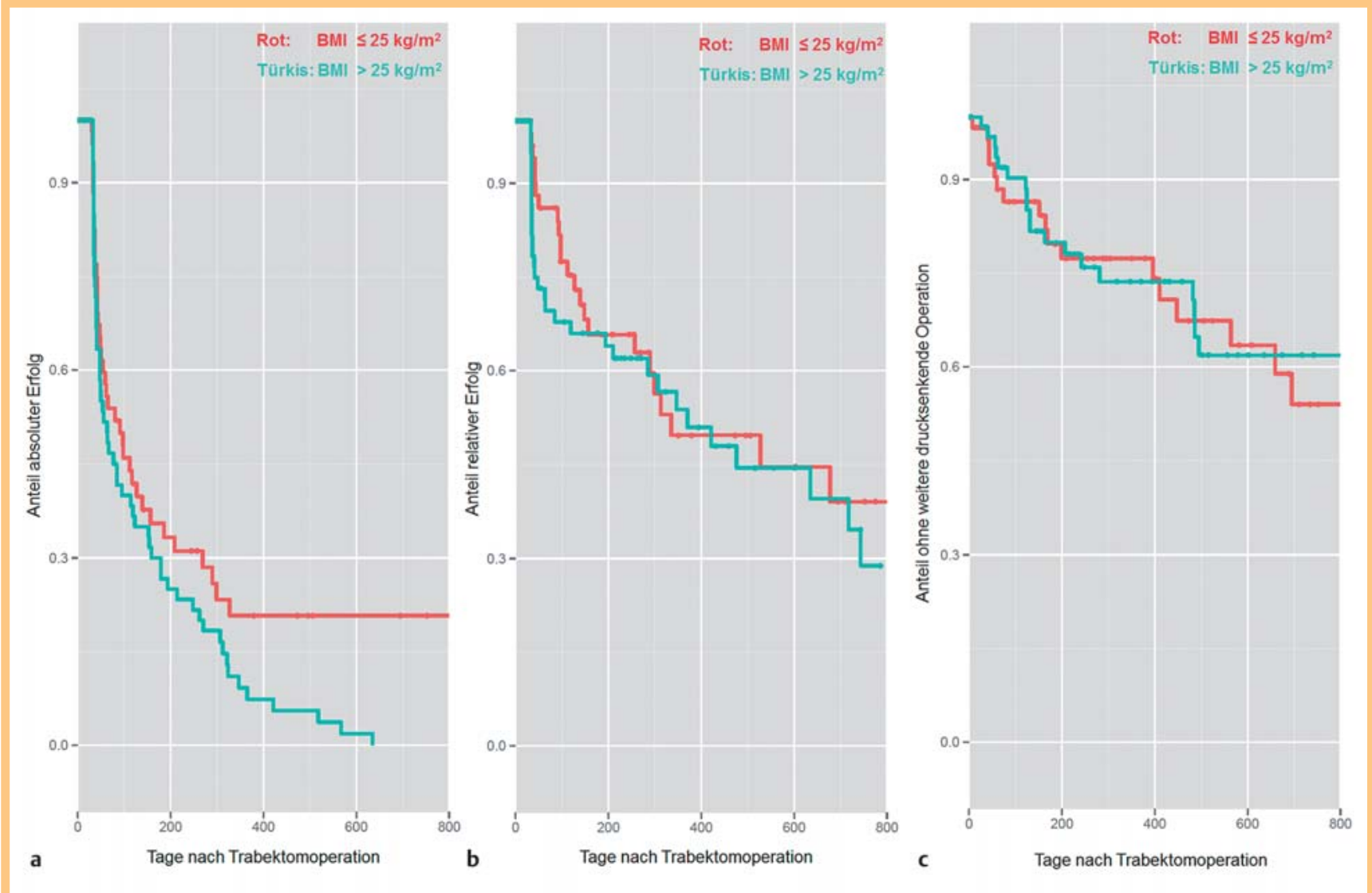

Abb. 1 Kaplan-Maier-Überlebensschätzung bezogen auf a absoluter Erfolg (20\% Drucksenkung vom Ausgangsdruck ohne drucksenkende Medikation). senkender Medikation). c Durchführung einer erneuten drucksenkenden b Relativer Erfolg ( $20 \%$ Drucksenkung vom Ausgangsdruck mit lokal druckOperation.

Tab. 2 Cox-Proportional-Hazards-Modell bezogen auf den absoluten Erfolg.

\begin{tabular}{|lll} 
& HR (Cl) & P \\
\hline BMI $>25 \mathrm{~kg} / \mathrm{m}^{2}$ & $1,54(1,03-2,34)$ & 0,04 \\
\hline Alter (in Jahren) & $0,99(0,97-1,01)$ & 0,53 \\
\hline $\begin{array}{l}\text { Anzahl der präoperativen } \\
\text { Augentropfen }\end{array}$ & $0,95(0,82-1,10)$ & 0,47 \\
\hline
\end{tabular}

HR: Hazard Ratio, P: p-Wert, Cl: 95\%-Konfidenzintervall. Ein BMl > 25 kg $/ \mathrm{m}^{2}$ erhöht das Risiko des Misserfolgs auf das 1,54-Fache des Normalgewichtigen.

\section{Ergebnisse}

Patienten mit normalem/niedrigem BMI zeigten eine bessere Prognose für eine Drucksenkung von mindestens $20 \%$ ohne zusätzliche Lokaltherapie nach Trabektomoperation. Im Cox-Modell hat die Gruppe mit einem BMI $>25 \mathrm{~kg} / \mathrm{m}^{2}$ ein statistisch signifikant schlechteres Ergebnis bezogen auf den absoluten Erfolg nach Trabektomoperation ( $\mathrm{p}=0,047,95 \%$-Konfidenzintervall: 1,03-2,34) (@ Tab. 2). Auf den relativen Erfolg sowie die Reoperationsrate nach Trabektomoperation hat der BMI keinen Einfluss. Hier unterscheiden sich die beiden Gruppen statistisch nicht signifikant voneinander ( $\bullet$ Abb. $\mathbf{1})$.

\section{Diskussion}

In unseren Untersuchungen zeigt sich ein statistisch signifikant negativer Einfluss von hohem BMI auf die Rate des absoluten Erfolgs nach Trabektomoperation, jedoch kein Einfluss auf den relativen Erfolg nach Trabektomoperation oder die Reoperationsrate. Ein schlechteres Ergebnis nach Trabektomoperation bei Übergewichtigen kann mit dem erhöhten episkleralen Venendruck [13] und damit erhöhtem Abflusswiderstand begründet werden. Kritisch zu beachten ist, dass in unserer retrospektiven Studie die Augendruckmessungen im Sitzen vorgenommen wurden. Da es im Liegen bei Übergewichtigen durch einen erhöhten intraabdominellen Druck zu einem Anstieg im episkleralen Venensystem kommt [8,14], könnte der Unterschied beider Gruppen im Liegen größer ausfallen. Jedoch zeigte Geloneck et al. [9], dass der BMI keinen signifikanten Einfluss auf die IOD-Änderung vom sitzenden zum liegenden Patienten hat, sodass dies auf unsere Ergebnisse vermutlich keinen Einfluss hat. Dieser negative Einfluss des hohen BMI auf den absoluten Erfolg nach Trabektomoperation scheint sich jedoch durch additive drucksenkende Lokaltherapie ausgleichen zu lassen, denn auf den relativen Erfolg hat der BMI bei unserer Untersuchung keinen Einfluss. Wenn der Patient nach der Operation bereit ist, weiter zu tropfen, kann man den BMI bei der klinischen Indikationsstellung zur Operation vernachlässigen. Bei Patienten mit multipler Tropfenunverträglichkeit spielt der BMI dahingegen eine Rolle, da der BMI auf den absoluten Erfolg (20\% Drucksenkung vom Ausgangsdruck ohne 
drucksenkende Medikation) einen negativen Einfluss hat. Der Umkehrschluss dahingegen, dass Patienten, um einen besseren absoluten Erfolg nach Trabektomoperation zu erreichen, abnehmen sollten, lässt sich aus unseren Daten nicht ziehen.

Auch auf die Reoperationsrate hat der BMI in unseren Untersuchungen keinen Einfluss. Dies unterstützt die Feststellung, dass es keinen Einfluss von BMI auf den relativen Erfolg gibt. Denn eine Indikation zur Reoperation ergibt sich nicht bei mit lokaler Drucksenkung gut eingestelltem Augendruck.

Ein weiterer Erklärungsansatz für das schlechtere absolute Ergebnis bei gleicher Reoperationsrate liegt in der besseren Drucktoleranz bei Patienten mit hohem BMI. Bei Patienten mit hohem BMI wurde ein höherer Liquordruck [15] sowie ein höherer Augendruck beobachtet $[4,16]$. Trotz des höheren Augendrucks haben Patienten mit erhöhtem BMI jedoch kein erhöhtes Glaukomrisiko $[4,16]$. Eine Erklärung hierfür könnte der geringere translaminäre Druckgradient zwischen zerebrospinaler Flüssigkeit und Augeninnendruck sein $[15,17,18]$. Berdahl et al. [19] führte eine retrospektive Analyse des Liquordrucks bei mehr als 30000 Individuen mit und ohne Glaukom durch. Sie fanden einen statistisch signifikant niedrigeren Liquordruck bei Patienten mit Glaukom unabhängig von der Ursache für die Liquorpunktion oder dem Alter. In einer linearen Regressionsanalyse zeigten sie einen signifikanten Zusammenhang von Cup-to-Disc-Ratio mit dem IOD, Liquordruck und translaminarem Druckgradienten. Dieser reduzierte translaminare Druckgradient bei Individuen mit hohem BMI könnte durch reduzierten Stress auf die einzelnen Sehnervenfasern in einem reduzierten Risiko für eine Sehnervenschaden resultieren [20], obwohl der Augendruck tendenziell höher liegt. Eine erneute Indikation zur Operation ergibt sich bei fehlender postoperativer Progression trotz schlechteren absoluten Erfolgs nicht. Obwohl die Patienten mit dem hohen BMI also einen geringeren absoluten Erfolg haben, müssen diese trotzdem nicht öfter reoperiert werden, da der Sehnerv bei Patienten mit hohem BMI nicht so vulnerabel ist.

Bei der Interpretation unserer Ergebnisse ist zu bedenken, dass es sich um eine relativ geringe Fallzahl handelt. Der negative Einfluss des hohen BMI auf den absoluten Erfolg ist statistisch nur knapp signifikant. Da es sich um eine retrospektive Studie handelt, fehlen prospektive Zielkriterien. So wurde die Indikation zur Reoperation klinisch, für jeden Patienten individuell festgelegt. Ob sich die Ergebnisse bestätigen lassen, muss in einer prospektiven Studie mit definierten Zielkriterien und größerer Fallzahl überprüft werden.

\section{Zusammenfassung}

Ein hoher BMI scheint einen negativen Einfluss auf die Rate des absoluten Erfolgs nach Trabektomoperation zu haben. Durch eine additiv lokal drucksenkende Therapie scheint dies jedoch ausgleichbar zu sein, sodass die Reoperationsrate in beiden Gruppen gleich liegt.

\section{Interessenkonflikt}

$\nabla$

Nein.

\section{Literatur}

1 Finucane MM, Stevens GA, Cowan MJ et al. National, regional, and global trends in body-mass index since 1980: systematic analysis of health examination surveys and epidemiological studies with 960 countryyears and 9.1 million participants. Lancet 2011; 377: 557-567

2 Haidar YM, Cosman BC. Obesity epidemiology. Clin Colon Rectal Surg 2011; 24: 205-210

3 Quigley HA, Broman AT. The number of people with glaucoma worldwide in 2010 and 2020. Br J Ophthalmol 2006; 90: 262-267

4 Ramdas WD, Wolfs RC, Hofman A et al. Lifestyle and risk of developing open-angle glaucoma: the Rotterdam study. Arch Ophthalmol 2011; 129: 767-772

5 Imai K, Hamaguchi M, Mori K et al. Metabolic syndrome as a risk factor for high-ocular tension. Int J Obes (Lond) 2010; 34: 1209-1217

6 Mori K, Ando F, Nomura H, Sato Y et al. Relationship between intraocular pressure and obesity in Japan. Int J Epidemiol 2000; 29: 661-666

7 Shiose $Y$. The aging effect on intraocular pressure in an apparently normal population. Arch Ophthalmol 1984; 102: 883-887

8 Bloomfield GL, Ridings PC, Blocher CR et al. A proposed relationship between increased intra-abdominal, intrathoracic, and intracranial pressure. Crit Care Med 1997; 25: 496-503

9 Geloneck MM, Crowell EL, Wilson EB et al. Correlation between intraocular pressure and body mass index in the seated and supine positions. J Glaucoma 2013; [Epub ahead of print] DOI: 10.1097/01.ijg. 0000435775.05032 .87

10 Johnson $M$. 'What controls aqueous humour outflow resistance?'. Exp Eye Res 2006; 82: 545-557

11 Fellman RL, Grover DS. Episcleral venous fluid wave: intraoperative evidence for patency of the conventional outflow system. J Glaucoma 2012; [Epub ahead of print] DOI: 10.1097/IJG.0b013e31827a06d8

12 Jordan JF, Wecker T, Van Oterendorp C et al. Trabectome surgery for primary and secondary open angle glaucomas. Graefes Arch Clin Exp Ophthalmol 2013; 251: 2753-2760

13 Jørgensen JS, Guthoff $R$. [The role of episcleral venous pressure in the development of secondary glaucomas]. Klin Monatsbl Augenheilkd 1988; 193: 471-475

14 Friberg TR, Sanborn G, Weinreb RN. Intraocular and episcleral venous pressure increase during inverted posture. Am J Ophthalmol 1987; 103: 523-526

15 Berdahl JP, Fleischman D, Zaydlarova J et al. Body mass index has a linear relationship with cerebrospinal fluid pressure. Invest Ophthalmol Vis Sci 2012; 53: 1422-1427

16 Leske MC, Connell AM, Wu SY et al. Risk factors for open-angle glaucoma. The Barbados Eye Study. Arch Ophthalmol 1995; 113: 918-924

17 Jonas JB, Wang $N$. Cerebrospinal fluid pressure and glaucoma. J Ophthalmic Vis Res 2013; 8: 257-263

18 Ren R, Wang N, Zhang $X$ et al. Cerebrospinal fluid pressure correlated with body mass index. Graefes Arch Clin Exp Ophthalmol 2012; 250: 445-446

19 Berdahl JP, Allingham RR, Johnson DH. Cerebrospinal fluid pressure is decreased in primary open-angle glaucoma. Ophthalmology 2008; 115: 763-768

20 Ren $R$, Zhang $X$, Wang $N$ et al. Cerebrospinal fluid pressure in ocular hypertension. Acta Ophthalmol 2011; 89: e142-e148 